\title{
Advanced Correlation Functionals: Application to Bulk Materials and Localized Systems
}

\author{
P. García-González, ${ }^{1,2}$ J. J. Fernández, ${ }^{1}$ Andrea Marini, ${ }^{2,3}$ and Angel Rubio ${ }^{2,4}$ \\ ${ }^{1}$ Departamento de Fúsica Fundamental, \\ Universidad Nacional de Educación a Distancia, \\ Apartado 60.141, E-28080 Madrid, Spain \\ ${ }^{2}$ European Theoretical Spectroscopy Facility (ETSF) \\ ${ }^{3}$ Istituto Nazionale per la Fisica della Materia e Dipartimento \\ di Fisica dell'Universitá di Roma "Tor Vergata", \\ Via della Ricerca Scientifica, I-00133 Roma, Italy \\ ${ }_{4}^{4}$ Departamento de Física de Materiales, Edificio Korta, \\ Universidad del País Vasco, Centro Mixto CSIC-UPV, \\ and Donostia International Physics Center (DIPC), \\ Avenida de Tolosa 71, E-20018 Donostia-San Sebastián, Spain
}

(Dated: June 15, 2007)

\begin{abstract}
Time-dependent density functional theory (TDDFT) is an efficient method to evaluate excitedstate properties of electron systems. However, it is not so well known that it also provides a very accurate prescription to obtain correlation energies by using the so-called adiabatic connection fluctuation dissipation theorem (ACFDT). In this paper we present a detailed study of the ACFDT performance in bulk solids and jellium clusters. These results confirm the reliability of the ACFDT scheme and pave the way to future applications where standard implementations of the Kohn-Sham density functional theory dramatically fail, in particular to weakly bound systems and van der Waals complexes.
\end{abstract}




\section{INTRODUCTION}

The Kohn-Sham (KS) implementation ${ }^{1}$ of density-functional theory (DFT) ${ }^{2}$ is one of the most powerful and used methods for electronic structure calculations in material science and quantum chemistry. In this scheme, the exact ground-state energy and electron density can be obtained self-consistently if the so-called exchange-correlation (XC) energy functional $E_{\mathrm{XC}}[n]$ was known. Since $E_{\mathrm{XC}}[n]$ contains information about the quantum many-body effects in the electron interaction, its actual (and unknown) expression is very complicated. However, and this is one of the main reasons of the popularity of KS-DFT, rather crude approximations to $E_{\mathrm{XC}}[n]$ depending explicitly on the electron density $n(\mathbf{r})$ often provide very accurate results at a moderate computational cost. Examples of such prescriptions are the local-density approximation $(\mathrm{LDA})^{1}$ and the generalized gradient approximation $(\mathrm{GGA})^{3,4}$ which, presently, constitute the methods of choice for practical applications of KS-DFT. However, this family of approaches may be reaching a limit of accuracy and a next generation of hybrid orbital functionals (see below) are taking more relevance nowadays.

The limitations of the LDA and GGA are obviously due to their local or semi-local nature which implies that they are extremely short-ranged. Thus they cannot describe at all the very nonlocal nature of electron-electron correlations and, for instance, van der Waals dispersion forces are completely out of the scope of the LDA and GGA. Another concomitant consequence of their simplicity is the appearance of self-interaction errors which, for instance, compromises their accuracy when dealing systems with localized electrons. More complicated XC nonlocal functionals, like the so-called averaged and weighted density approximations, ${ }^{5-9}$ do not provide systematic improvements upon LDA and GGA while being computationally much more demanding.

Orbital-dependent (OD) XC functionals, that is, functionals that depend implicitly on the electron density through the Kohn-Sham orbitals, are the natural next step towards the formulation of DFT prescriptions with chemical accuracy. The obvious advantage is that they provide a description of the electron-electron interaction at a much deeper level than conventional density-based functionals. The main drawback is the increasing numerical cost. However, this next generation of $\mathrm{XC}$ functionals can be very competitive if compared with exact methods like quantum Monte Carlo or full configuration interaction. Under this perspective, OD functionals may be seen as a more flexible alternative to quantum chemistry 
techniques like Møller-Pleset perturbation theory and coupled cluster methods. The novel meta-GGA functionals proposed by Perdew and coworkers ${ }^{10-12}$ are the simplest examples of OD functionals. In this paper, however, we will focus on more sophisticated approaches that incorporates exactly the exchange energy functional. Namely,

$$
E_{\mathrm{XC}}[n] \simeq E_{\mathrm{X}}^{\mathrm{EXX}}[n]+E_{\mathrm{C}}[n],
$$

where $E_{\mathrm{C}}[n]$ is an approximation to the correlation energy functional and $E_{\mathrm{X}}^{\mathrm{EXX}}[n]$ is the exact exchange (EXX) energy functional given in terms of the occupied KS orbitals $\phi_{n}(\mathbf{r})$ :

$$
E_{\mathrm{X}}^{\mathrm{EXX}}[n]=-2 \times \frac{1}{2} \sum_{n m}^{\mathrm{occ}} \int d^{3} \mathbf{r} d^{3} \mathbf{r}^{\prime} \frac{c_{n m}(\mathbf{r}) c_{m n}\left(\mathbf{r}^{\prime}\right)}{\left|\mathbf{r}-\mathbf{r}^{\prime}\right|} .
$$

where $c_{n m}(\mathbf{r})=\phi_{n}^{*}(\mathbf{r}) \phi_{m}(\mathbf{r})$. The factor 2 appears as a result of the sum over spin-degrees of freedom (we will restricted ourselves to spin-unpolarized systems and use Hartree atomic units throughout the paper unless otherwise specified). Note that (2) has the same form that the well-known Hartree-Fock (HF) exchange, except that the KS orbitals are used instead of the HF ones. Therefore, the correlation energy is the only term that must be approximated in this EXX-based KS prescription. Nonetheless, the XC functionals given by (1) misses one of the reasons of the success of LDA (and to a lesser extent of GGA): the systematic cancellation of errors between the exchange and correlation counterparts. As a consequence, implementations of (1) with full predictive power require very accurate approximations to the correlation energy.

An interesting route is the use of many-body theory which defines a perturbative formulation of the correlation energy in terms of the KS orbitals and eigenenergies, ${ }^{13-15}$ much akin to the standard Møller-Pleset expansion. Another option is the use of the following exact expression for the correlation energy functional:

$$
\begin{aligned}
E_{\mathrm{C}}[n]= & -\int_{0}^{\infty} \frac{d u}{2 \pi} \int_{0}^{1} d \lambda \int d^{3} \mathbf{r} d^{3} \mathbf{r}^{\prime} \frac{1}{\left|\mathbf{r}-\mathbf{r}^{\prime}\right|} \\
& \times\left[\chi_{\lambda}\left(\mathbf{r}, \mathbf{r}^{\prime} ; \mathrm{i} u\right)-\chi_{0}\left(\mathbf{r}, \mathbf{r}^{\prime} ; \mathrm{i} u\right)\right]
\end{aligned}
$$

known as adiabatic connection fluctuation-dissipation theorem (ACFDT). ${ }^{16,17}$ Here, $\chi_{\lambda}\left(\mathbf{r}, \mathbf{r}^{\prime} ; \mathrm{i} u\right)$ is the imaginary-frequency density response of a fictitious system of electrons interacting through a scaled Coulomb potential $\lambda / r_{12}$ and whose ground-state density equals the actual one. Then, $\chi_{0}\left(\mathbf{r}, \mathbf{r}^{\prime} ; i u\right)$ is the response function of the fictitious non-interacting KS system:

$$
\chi_{0}\left(\mathbf{r}, \mathbf{r}^{\prime} ; \mathrm{i} u\right)=2 \times \sum_{n m} \frac{\left(f_{n}-f_{m}\right) c_{n m}(\mathbf{r}) c_{m n}\left(\mathbf{r}^{\prime}\right)}{\mathrm{i} u+\left(\varepsilon_{n}-\varepsilon_{m}\right)}
$$


where $f_{n}(0$ or 1$)$ are Fermi occupation numbers and $\varepsilon_{n}$ the KS eigenenergies. The interacting response $\chi_{\lambda}$ can be evaluated in the framework of time-dependent density functional theory $(\mathrm{TDDFT})^{18-20}$ by solving the Dyson-like equation

$$
\widehat{\chi}_{0}(\mathrm{i} u)=\left(\widehat{1}-\widehat{\chi}_{0}(\mathrm{i} u)\left[\lambda \widehat{w}+\widehat{f}_{\mathrm{XC}, \lambda}(\mathrm{i} u)\right]\right) \widehat{\chi}_{\lambda}(\mathrm{i} u)
$$

where the usual matrix operations are implied. $\widehat{w}$ is the bare Coulomb interaction and $\widehat{f}_{\mathrm{XC}, \lambda}(\mathrm{i} u)$ is the dynamical XC kernel of the fictitious system with the scaled interaction $\lambda \widehat{w}$. Thus, the evaluation of the correlation energy only relies on the approximations made to $\widehat{f}_{\mathrm{XC}, \lambda}$. Also note that under this formulation the ground-state energy and the optical properties of the electron system (related to the neutral excitations in the system) are treated under the same framework, which incorporates higher order electron interactions whose treatment has shown to be mandatory in ab-initio calculations of spectroscopic properties of extended systems. ${ }^{19,20}$

As anticipated, the implementation of this ACFDT scheme is much more computationally demanding than usual KS methods. However it is a promising ab-initio total-energy method due to a number of reasons. First, exchange and correlation are treated at the same level. Second, ACFDT accounts for van der Waals forces,${ }^{21-23}$ thus being a suitable approach for an unified treatment of electron-electron interactions with different spacial ranges, a situation that appears in bundled nanotubes, polymer crystals and, in general, sparse systems. Third, by construction there are not self-interaction errors in the exchange part whereas they do not seem to be very serious in the correlation term and might be systematically reduced by choosing a proper kernel. Finally, it serves as the starting point of further simplifications aimed for implementation in very complex systems ${ }^{24,25}$ at an affordable computational cost. On the other hand, a first evident problem of the ACFDT is the dependence of the results on the choice of the XC kernel, which is especially relevant when considering total correlation energies. ${ }^{26}$ However, there are some evidences that such a choice is not so critical for the evaluation of structural properties, which are related to energy differences in isoelectronic systems..$^{27,28}$ Nonetheless, this point is still an open issue, ${ }^{29}$ and some interesting attempts directed to find an optimal XC kernel for correlation energy calculations have been already presented. ${ }^{30-32}$ Secondly, the evaluation of the ACFDT correlation potential $v_{\mathrm{C}}(\mathbf{r})$ is a formidable task, ${ }^{33-35}$ much more demanding that the calculation of the EXX potential $v_{\mathrm{X}}(\mathbf{r}),{ }^{36,37}$ which is presently carried out routinely in both molecular ${ }^{38,39}$ 
and extended systems. ${ }^{40,41}$ In fact, the self-consistent evaluation of the ACFDT $v_{\mathrm{C}}(\mathbf{r})$ has been only implemented for model electron systems, ${ }^{42,43}$ atoms,${ }^{44}$ and simple bulk crystalline solids. ${ }^{45}$ Thus, almost all the applications of the ACFDT scheme made so far ${ }^{29-32,46-53}$ have neglected any attempt of self-consistency, and the correlation energy is evaluated using LDA, GGA or EXX Kohn-Sham wavefunctions and eigenenergies as an input, as commonly done in excited-state $a b$-initio calculations. ${ }^{20}$ This prescription will be followed in this paper as well, where we will present detailed ACFDT results for two rather simple, but very different, systems: compact bulk structures (silicon and sodium chloride) and jellium metal clusters.

For simple bulk systems, LDA already shows a very good performance. Hence, it is not expected that the sophisticated EXX/ACFDT XC functional will lead to significant differences with respect to the LDA results. However, it is important to show that this good performance is not compromised by the more developed ACFDT functional. Then, our main goal will be the detailed discussion of the implementation of the ACFDT to extended crystalline systems. Since many-body effects in the correlation energy are not going to be critical, we will use the simplest ACFDT prescription and neglect the $\mathrm{XC}$ kernel in the evaluation of the interacting density response (i.e. $\chi$ is obtained in a Random Phase Approximation [RPA] fashion). As it is known, RPA accounts for long-range correlation effects but the description of short-range ones is very poor. Thus, RPA gives too deep correlation holes and too large (in absolute value) correlation energies. Fortunately, these short-range effects can be effectively modelled by an LDA or GGA correction term ${ }^{27,28}$ :

$$
\begin{aligned}
E_{\mathrm{C}}[n] & \simeq E_{\mathrm{C}}^{\mathrm{RPA}}[n]+\Delta E_{\mathrm{C}}[n] \\
& =E_{\mathrm{C}}^{\mathrm{RPA}}[n]+\int d^{3} \mathbf{r} n(\mathbf{r}) \varepsilon_{\mathrm{cor}}(n(\mathbf{r}), \nabla n(\mathbf{r})),
\end{aligned}
$$

where $\varepsilon_{\text {cor }}$ is a function of the local density and its gradient. ${ }^{27,28}$ As we will see this hybrid scheme, often called RPA+, suffices to obtain very accurate correlation energies.

On the contrary, jellium metal cluster are examples of small localized systems where the elimination of self-interaction errors is required to obtain reliable results. Furthermore, by increasing the size of the cluster we approach the homogeneous electron gas limit and hence, in spite of the simplicity of these systems, different correlation regimes appear. The existence of accurate benchmark quantum Monte Carlo calculations ${ }^{54}$ allows us to assess not only the performance of the RPA + correlation functional, but also the one corresponding to the evaluation of the response function using different approaches to the XC kernel. 
The outline of this article is as follows. In section 2 we will present a detailed description of our implementation of the ACFDT scheme for crystal solids, applied recently ${ }^{53}$ to the problem of layer-layer interactions in laminar systems. Section 3 contains the results and discussion for bulk $\mathrm{Si}$ and $\mathrm{NaCl}$, whereas section 4 is devoted to the ACFDT results for metal clusters, covering different ranges of densities and electron number. The corresponding conclusions and perspectives will close this paper.

\section{THE ACFDT SCHEME FOR SOLIDS}

Our implementation of the ACFDT correlation functional for crystalline solids is built on the long-standing experience gained in the last years in ab-initio TDDFT calculations ${ }^{20}$ as reflected by the existence of a number of efficient computational tools. Specifically, KS wavefunctions and operators are represented in a planewave basis set and core electrons are approximately described using standard non-local pseudopotentials. ${ }^{55}$ Hence, KS wavefunctions and energies are labelled by a band index $n$ and a vector $\mathbf{k}$ belonging to the first Brillouin zone (BZ). Due to the periodicity of the system, the reciprocal space representation of the response functions takes the form $\chi_{\lambda}\left(\mathbf{k}+\mathbf{G}_{1}, \mathbf{k}+\mathbf{G}_{2} ; \mathbf{i} u\right)$, where $\mathbf{G}$ is a vector of the reciprocal lattice. The ACFDT correlation energy per volume unit is then given by

$$
\frac{E_{\mathrm{C}}}{V}=-\int_{0}^{1} d \lambda \int_{0}^{+\infty} \frac{d u}{2 \pi} \int_{\mathrm{BZ}} d^{3} \mathbf{k} \sum_{\mathbf{G}} e_{\mathrm{C}}(\mathbf{k}, \mathbf{G}, u, \lambda)
$$

with

$$
e_{\mathrm{C}}(\mathbf{k}, \mathbf{G}, u, \lambda)=\frac{4 \pi}{|\mathbf{k}+\mathbf{G}|^{2}} \Delta \chi_{\lambda}(\mathbf{k}+\mathbf{G}, \mathbf{k}+\mathbf{G} ; \mathbf{i} u)
$$

and $\Delta \chi_{\lambda}=\chi_{\lambda}-\chi_{0}$. The KS response $\chi_{0}$ is calculated from well-converged KS wavefunctions and energies evaluated using the ABINIT package. ${ }^{56}$ The interacting responses $\chi_{\lambda}$ are then obtained for each vector $\mathbf{k}$ in the Brillouin zone, imaginary frequency $u$, and coupling parameter $\lambda$ solving (5) using the SELF code, ${ }^{57}$ where the expressions (7-8) are implemented as well.

At a first glance, this implementation seems to be straightforward, but there are some technical issues that must be solved in order to have a stable and easy to converge numerical procedure. First, the dependences of the function $e_{\mathrm{C}}(\mathbf{k}, \mathbf{G}, u, \lambda)$ defined in (8) on the coupling constant $\lambda$ and the imaginary frequency $u$ are very smooth. Thus, both integrals can be carried out in a very efficient way using Gauss-Legendre (GL) samplings. For $\lambda$, a six-point 
GL grid is enough for our purposes, whereas the frequency integral is done using two GL grids, each typically comprising 18-24 points. The first one lies in the range $\left[0, u_{1}\right]$, where $u_{1}$ is of the order of the plasmon frequency associated to the mean density of the system, and the second one lies in the interval $\left[u_{1}, u_{\max }\right]$, where $u_{\max }$ is a frequency cutoff. This procedure allows an accurate evaluation of the contributions from both small and large imaginary frequencies. The convergence with respect the critical parameter $u_{\max }$ is carefully checked, but it does not pose any major problem.

Much more delicate is the convergence versus: i) the sampling of the BZ (determined by the number of special $\mathbf{k}$ vectors, $N_{k}$, in the irreducible wedge of the BZ), ii) the number of reciprocal lattice vectors $N_{G}$ (i.e. the energy cutoff in reciprocal space), and iii) the number of bands $N_{b}$ used to evaluate the KS response function (remember that $\chi_{0}$ contains contributions from occupied and unoccupied states). Unfortunately, the common practice in electronic structure calculations that fixes the energy cutoff for all geometries (and, hence, defines a different $N_{G}$ for each geometry) does not work in the present case. The convergence of $E_{\mathrm{C}}$ versus the number of bands is very slow and, what is more important, the convergence rate depends very sensitively on the considered geometry. Furthermore, the required numerical effort increases dramatically with the number of $\mathbf{k}$-points. All this prevents us from having reasonably converged energy differences and, as a consequence, the evaluation of structural properties is completely impossible in this way.

These problems can be circumvented since, for a fixed sampling of the BZ, the planewave representation allows a systematic simultaneous convergence with respect to $N_{G}$ and $N_{b}$. Namely, regardless the energy cutoff, the number of bands used in the evaluation of the KS response function is imposed by the expression $N_{b}=\left(N_{G}-1\right) / \alpha$, where $\alpha$ is an integer (typically equal to two, although we have checked that the same final results are obtained using $\alpha=3$ and 4 ). As we may see in Figure 1 , the convergence with respect $N_{G}$ is very well defined, and it is easy to obtain the infinite $N_{G}$ and $N_{b}$ limit of the correlation energy as long as the number of $\mathbf{k}$ points is small. However, this procedure is not feasible for finer samplings of the BZ. Fortunately, the differences induced in the correlation energy by increasing $N_{k}$ are very insensitive to the number of $G$ vectors used in the calculation (see the inset in Figure 1). This suggests a well-defined procedure. First, using a coarse BZ sampling, the correlation energy is converged by increasing simultaneously $N_{G}$ and $N_{b}$. Second, using the results corresponding to the largest $N_{G}$ s, the infinite limit result is obtained by extrapolation 


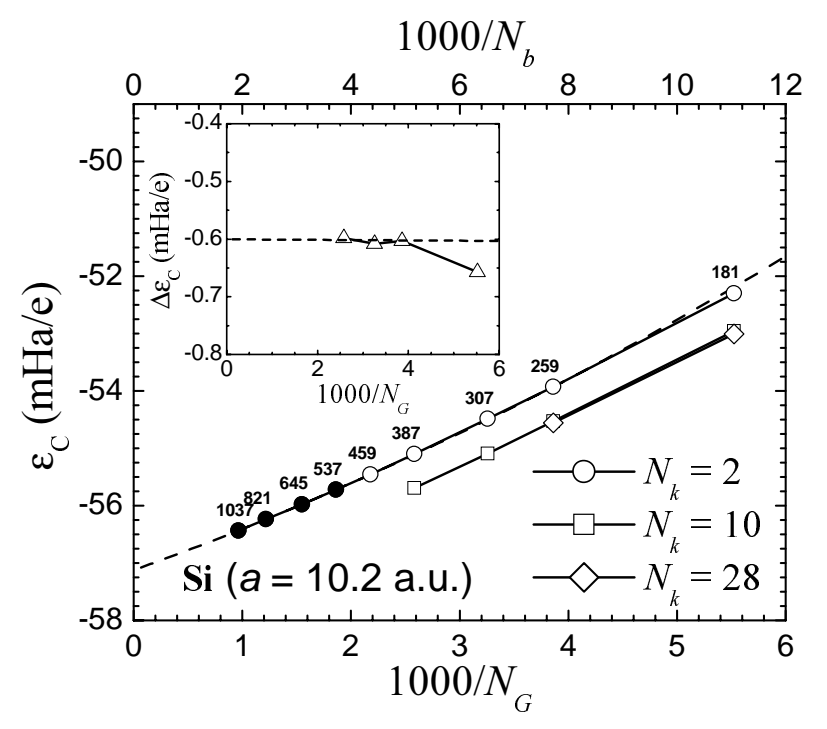

Figure 1. Analysis of the convergence of the RPA correlation energy for bulk silicon (lattice parameter $a=10.2$ a.u.). For a coarse sampling of the Brillouin zone with just two special points (hollow and solid circles), the limit corresponding to an infinite number of bands and $\mathbf{G}$ vectors can be easily extrapolated from the values indicated with solid symbols. The reliability of the fitting curve (dashed line) is evident since it fits other values not used in the extrapolation. For a finer BZ sampling with 10 special points (squares), the change on the correlation energy with respect to the previous one converges very quickly with the number of $\mathbf{G}$ vectors, as represented in the inset. Finally, it can be

seen that $N_{k}=10$ provides full convergence since the inclusion of more $\mathbf{k}$ vectors (rhombuses) does not lead to appreciable variations in the correlation energy. Note that the numerical error in the final result $(-57.8 \pm 0.2 \mathrm{mHa} / \mathrm{e})$ is mostly due to the uncertainties in the extrapolation procedure. The number of $\mathbf{G}$ vectors used in the calculations are displayed in the figure.

ensuring that the extrapolating curve also fits the rest of the values. Finally, the error due to the coarse BZ sampling is determined by comparing the correlation energy obtained using more $\mathbf{k}$ points, but evaluated with a relatively small number of reciprocal lattice vectors. By doing this we are fully confident that the absolute correlation energy for a given geometry is converged within a numerical error less than $0.2 \mathrm{mHa}$ per electron, whereas for correlation energy differences, such an error turns out to be of the order of $0.05 \mathrm{mHa}$ per electron.

Finally, is worth mentioning a few words about exact exchange. When calculating the 
EXX energy, the only critical parameter is $N_{k}$, because only occupied wave functions enters into the evaluation and the $N_{G}$-convergence is quite fast. In fact, $E_{\mathrm{X}}^{\operatorname{EXX}}$ converges rather slowly with respect to the BZ sampling, and a relatively large number of special $\mathbf{k}$ points is required. Nevertheless, this part of the calculation is inexpensive compared with the ACFDT one and, furthermore, the convergence can be sped up using random integration techniques over the BZ zone. ${ }^{58}$ The absolute numerical errors in the EXX energies are of the same order of magnitude than the ACFDT correlation ones, although we have to bear in mind that the exchange energy is often several times larger than the correlation one.

\section{RESULTS FOR BULK STRUCTURES}

The methodology described in the previous section allows us to calculate the exchangecorrelation energy for crystalline solids at different geometries, thus giving us access to a fully microscopic evaluation of structural properties, including selected lattice dynamics using a frozen-phonon approximation. As mentioned before, this scheme has been already applied to the calculation of equilibrium structural properties of a layered material (hexagonal boronnitride). ${ }^{53}$ This is a system where long-range van der Waals forces coexist with short-ranged covalent ones, thus being a perfect scenario where the ACFDT, under the RPA+ approach, shows its full capacity. In this section we will describe the results for bulk silicon (the paradigmatic example of $s p$ insulator) and, very briefly, for $\mathrm{NaCl}$ (an example of ionic solid). We can anticipate that there will be marginal differences between the LDA and the EXX/RPA+ results. Those differences are going to be less important than, for instance, the choice of the pseudopotential used in the calculations. Nevertheless, these calculations illustrate very well the known cancellation of errors between exchange and correlation in the LDA prescription and the robustness of the EXX/RPA+ approximation.

The EXX and the RPA+ correlation energies per unit cell for bulk silicon at different lattice constants are presented and compared with the LDA counterparts in Figure 2. The most noticeable features are the discrepancies between exchange and correlation energies if considered separately, which reflects the well known LDA underestimation (overestimation) of the absolute value of the exchange (correlation) energy. However, both LDA errors compensate each other and the total LDA and EXX/RPA + XC energies are practically the same. A similar cancellation appears if we consider the lattice parameter dependence of 


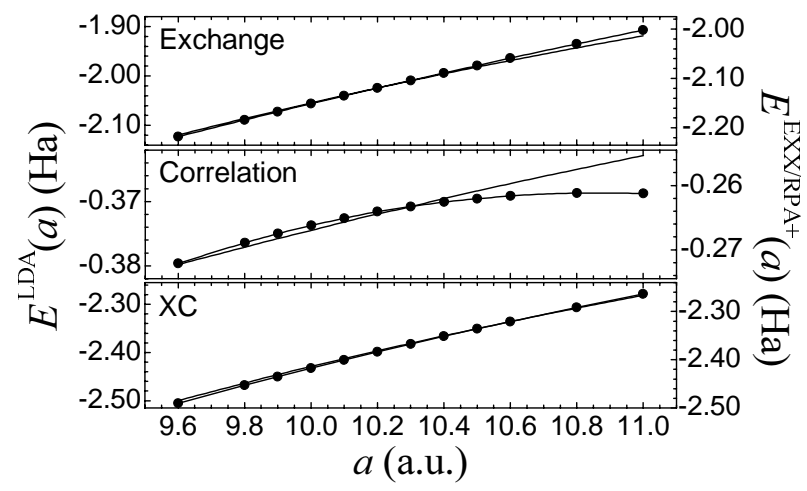

Figure 2. Exchange and correlation energies per unit cell for bulk silicon as functions of the lattice parameter $a$ using EXX/RPA+ (solid lines with circles) and LDA (solid line). Note that the energy scale for the correlation is much smaller than the one corresponding to exchange and exchange-correlation. The LDA greatly overestimates both the absolute value of the correlation energy and its variation with the lattice constant. The behavior of the LDA exchange is the opposite if compared with the EXX results.

the $\mathrm{XC}$ energy, although it is worth emphasizing the very different behavior exhibited by the LDA correlation energy if compared with the RPA+ one. However, exchange dominates upon correlation in bulk Si and this discrepancy will not affect too much to the final structural properties. We must mention the LDA correction term $\Delta E_{\mathrm{C}}[n]$ has an almost negligible dependence on the lattice parameter. In other words, the RPA and RPA+ correlation curves are practically parallel and, as a consequence, the inclusion of $\Delta E_{\mathrm{C}}[n]$ simply shifts rigidly the total energy, without affecting the rest of the equilibrium properties of bulk Si, but providing total energies as accurate as LDA.

The variation of the total energy per unit cell with respect the lattice constant $a$ is represented in Figure 3. We may see that the inclusion of many-body effects via the EXX/RPA+ leads to a marginal reduction (less than $0.5 \%$ ) of the equilibrium lattice constant if compared with the LDA one (10.17 a.u.). Similarly, the bulk modulus is unaffected within the unavoidable numerical error bars. At a first glance, this small correction goes in the wrong direction, since the experimental lattice constant $\left(a_{\exp }=10.26\right.$ a.u. $)$ is roughly a $1 \%$ larger than the LDA one. Nevertheless, we must point out that: i) our EXX/RPA+ implementation is not self-consistent, but evaluated using LDA wavefunctions, ii) the RPA+ approximation to the exact correlation functional is the simplest one within the ACFDT scheme, iii) we 


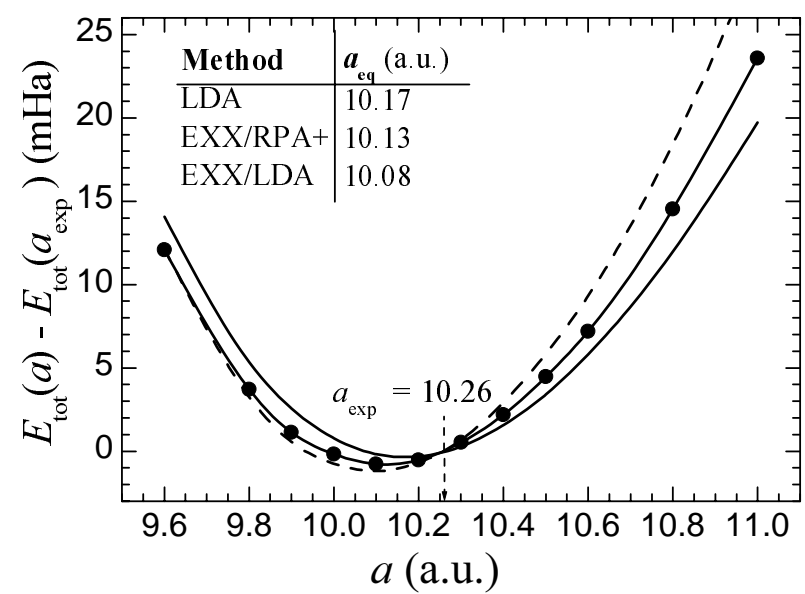

Figure 3. Total energy per unit cell for bulk silicon as a function of the lattice parameter $a$ obtained from EXX/RPA+ (solid line with circles), LDA (solid line), and EXX/LDA (dashed line). In the EXX/LDA, the exchange energy is calculated exactly and the correlation approximated using the LDA. All the calculations have been performed using the same pseudopotential. Note that the EXX/LDA lacks the typical LDA cancellation of errors. The corresponding equilibrium lattice constants are included within the figure. The experimental value, $a_{\exp }$ is indicated as a reference.

are working under a pseudopotential approximation. Very likely, the first point is going to be the least important. It is known for a long time ${ }^{59,60}$ that for bulk $s p$ semiconductors, the shape of the LDA XC potential is very similar than the one corresponding to the local $\mathrm{XC}$ potential obtained from the linearized form of the Sham-Schlüter equation, ${ }^{61,62}$ which formally corresponds to the EXX/RPA potential. ${ }^{33,34,63}$ This overall coincidence has been recently confirmed by state-of-the-art calculations. ${ }^{45}$ The second point should deserve further attention, but it is very likely that for this simple compact $s p$ structure the inclusion of effects beyond the RPA via appropriate XC kernels is going to have a marginal influence on the final results. Thus, we can conclude that the pseudopotential approximation itself is the most important source of error in our calculation. At this point, we have to mention that Miyake $e t a l^{52}$ presented a few years ago a prospective study of the RPA correlation energy for bulk systems in an all-electron picture. As can be easily inferred by the discussion in section 2 (restricted to the much simpler pseudopotential picture), the numerical difficulties that these authors had to face were formidable. Hence, their quantitative results must be taken with caution, although their main qualitative conclusions agrees very well with the 


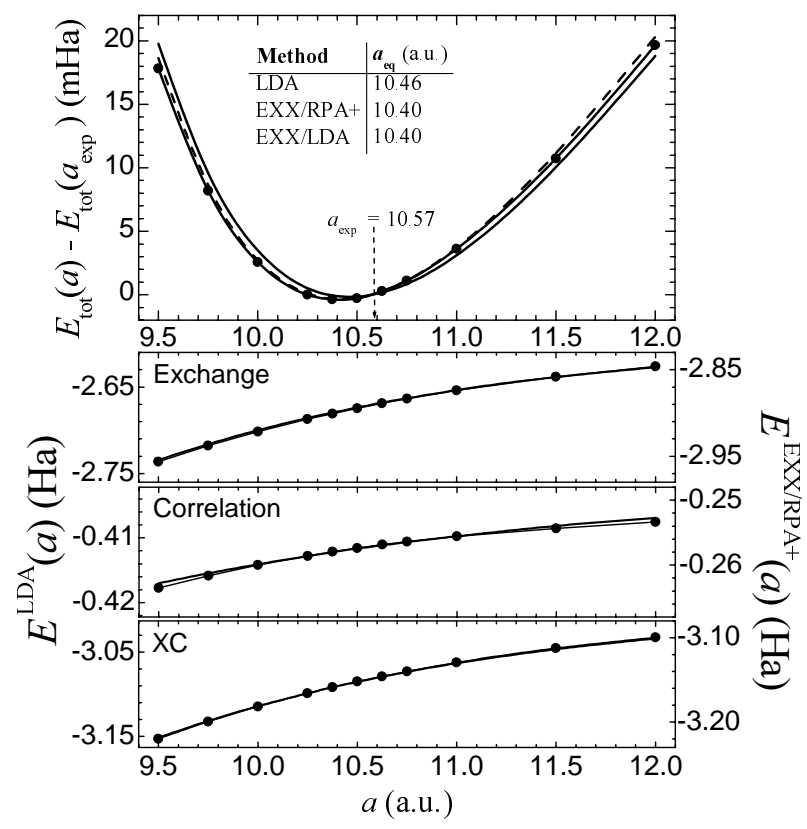

Figure 4. $\quad$ Upper panel: same as Figure 3 for bulk NaCl. Lower panels: same as Figure 2 for bulk NaCl. The many-body effects included in the RPA+ correlation can be only appreciated far from the equilibrium geometry, which explains why the EXX/LDA and EXX/RPA+ lattice constants are the same.

ones obtained from our well converged pseudopotential-based results.

Finally, the EXX/RPA+ description of the XC effects in $\mathrm{NaCl}$ leads to similar conclusions. As can be seen in the upper panel of Figure 4, the LDA and EXX/RPA+ equilibrium properties are practically the same. Moreover, the discrepancies in the exchange and correlation energy curves are even smaller than in the case of bulk $\mathrm{Si}$. In fact, the substitution of the LDA correlation by the RPA+ one does not change the equilibrium lattice constant and the mentioned small discrepancies are mainly due to the different treatment of exchange.

\section{APPLICATION TO JELLIUM CLUSTERS}

Jellium clusters, where the positive ions are modelled by an uniform spherical positive background of density $n_{\mathrm{B}}$, have been used extensively in the past to study the experimental properties of alkali metal clusters. ${ }^{64,65}$ This approach is presently superseded by modern electronic structure calculations, although jellium clusters are still useful to assess advanced ab-initio theories. In this model, the cluster is fully characterized by the number of electrons 
$N$ and the mean background density $n_{\mathrm{B}}$. Since the system is neutral, the radius of the jellium sphere is $R_{\mathrm{B}}=N^{1 / 3} r_{\mathrm{s}}, r_{\mathrm{s}}=[3 /(4 \pi n)]^{1 / 3}$ being the so-called Wigner radius. Hence, the $N$ electrons move under the action of an electrostatic potential which is harmonic if $r \leq R_{\mathrm{B}}$ and Coulombic if $r \geq R_{\mathrm{B}}$.

As commented in the Introduction, there are accurate quantum Monte Carlo results for closed-shell jellium clusters ${ }^{54}$ which correspond to $N=2,8,18,20,34,40,58, \ldots$ at least in the range of metallic densities. Because of the closed-shell configuration, the electronic ground-state is nondegenerate and exhibits spherical symmetry. This enormously simplifies not only the implementation of the KS equations, but also the evaluation of the EXX and ACFDT correlation energies which can be carried out at a low computational cost. Therefore, this simple model is very suited to assess the performance of different implementations of the ACFDT scheme. Note that the problems that plague its application to real extended materials (as we have seen in section 2) and localized molecular systems ${ }^{29}$ are now completely absent since we can easily reach full numerical convergence.

Because of the spherical symmetry, the KS wave functions take the familiar form $\phi(\vec{r})=$ $R_{n \ell}(r) \mathcal{Y}_{\ell}^{m}(\Omega), R_{n \ell}$ being the radial wavefunction and $\mathcal{Y}_{\ell}^{m}(\Omega)$ an spherical harmonic. The density response $\chi_{\lambda}$ (and, in general, any involved operator) can be written as a Legendre expansion:

$$
\chi_{\lambda}\left(\mathbf{r}, \mathbf{r}^{\prime} ; \mathrm{i} u\right)=\sum_{L=0}^{\infty} \chi_{\lambda}^{(L)}\left(r, r^{\prime} ; \mathrm{i} u\right) \mathcal{P}_{L}(\cos \gamma)
$$

where $\mathcal{P}_{L}$ is the $L$-th order Legendre polynomial and $\gamma$ the angle formed by $\mathbf{r}$ and $\mathbf{r}^{\prime}$. $\chi_{0}^{(L)}\left(r, r^{\prime} ; \mathrm{i} u\right)$ can be readily expressed as a function of products of occupied $(n \ell)$ and unoccupied $\left(n^{\prime} \ell^{\prime}\right)$ radial wavefunctions, KS eigenergies, and Clebsch-Gordan coefficients in such a way that only the wavefunction products with $\left|\ell-\ell^{\prime}\right| \leq L \leq\left|\ell+\ell^{\prime}\right|$ enter into the evaluation of $\chi_{0}^{(L)}$. Unbounded unoccupied states are discretized by imposing infinite wall boundary conditions at a $r_{\max } \gg R_{\mathrm{B}}$. In our calculations, $r_{\max } \sim 10 R_{\mathrm{B}}$ suffices. Dyson's equation (5) is solved for each $L$-component separately, and the solution can be done either in real space or in a matrix representation. As we can see, the simplicity of the system reflects on the fact that the original three-dimensional problem can be effectively separated in $L$ decoupled one-dimensional ones. Finally, the integrations over the coupling constant $\lambda$ and the imaginary frequency are performed following the method already described in section 2 . We have to mention that the whole procedure could be made even simpler by following the 
implementation proposed by Furche. ${ }^{29,48}$ Moreover, the sum over unoccupied states could be circumvented by solving the Schrödinger-like equation obeyed by the one-electron KS Green's function, whose knowledge allows to obtain the KS density response.

Our first aim is the critical discussion of the performance of the RPA+ correlation functional for localized systems. We will also pay attention to more elaborated ACFDT prescriptions based on the evaluation of the density response including local field effects (i.e., corrections beyond RPA) using suitable XC nonlocal kernels. We will restrict ourselves to the well-known Petersilka-Gossman-Gross (PGG) kernel ${ }^{66}$ and to a recent energy-optimized nonlocal Hubbard-like kernel (OHU) proposed by Jung et al.$^{32}$ The PGG kernel

$$
f_{\mathrm{XC}, \lambda}^{\mathrm{PGG}}\left(\mathbf{r}, \mathbf{r}^{\prime} ; \omega\right)=-\frac{2 \lambda}{\left|\mathbf{r}-\mathbf{r}^{\prime}\right|} \frac{\left|\sum_{n}^{\mathrm{occ}} \phi_{n}(\mathbf{r}) \phi_{n}^{*}\left(\mathbf{r}^{\prime}\right)\right|^{2}}{n(\mathbf{r}) n(\mathbf{r})}
$$

is a static model which has the advantage of being the exact exchange-only kernel for a system with two electrons (and the exact XC kernel for a single electron system). Therefore, and that is the reason of this choice, it is in principle suitable to deal in an approximate manner the spurious self-interaction that appears in the response function for few electron systems. On the contrary, the OHU nonlocal kernel has a completely different physical motivation. It has the form

$$
f_{\mathrm{XC}, \lambda}^{\mathrm{OHU}}\left(\mathbf{r}, \mathbf{r}^{\prime} ; \omega\right)=\lambda^{2} F_{\mathrm{XC}}^{\mathrm{hom}}\left(\lambda \overline{r_{\mathrm{s}}}\left(\mathbf{r}, \mathbf{r}^{\prime}\right), \lambda\left|\mathbf{r}-\mathbf{r}^{\prime}\right|\right)
$$

where $\overline{r_{\mathrm{s}}}\left(\mathbf{r}, \mathbf{r}^{\prime}\right)=\left[3 /\left(4 \pi \sqrt{n(\mathbf{r}) n\left(\mathbf{r}^{\prime}\right)}\right)\right]^{1 / 3}$ is an effective Wigner radius and $F_{\mathrm{XC}}^{\text {hom }}\left(r_{\mathrm{s}}, r\right)$ is a static nonlocal approximation to the $\mathrm{XC}$ kernel of the homogenous electron gas (HEG). Such a kernel is given by

$$
F_{\mathrm{XC}}^{\mathrm{hom}}\left(r_{\mathrm{s}}, r\right)=\frac{\kappa\left(r_{\mathrm{s}}\right) \beta^{2}\left(r_{\mathrm{s}}\right)}{4 \pi} \frac{\exp \left(-\beta\left(r_{\mathrm{s}}\right) r\right)}{r}
$$

where $\kappa\left(r_{\mathrm{s}}\right)=d^{2}\left(n \varepsilon_{\mathrm{C}}\right) / d n^{2}\left(n\right.$ and $\varepsilon_{\mathrm{C}}$ being the HEG electron density and correlation energy per particle, respectively) and

$$
\beta\left(r_{\mathrm{s}}\right)=\left(\frac{9 \pi}{4}\right)^{1 / 3} \sqrt{\frac{100+5 r_{\mathrm{s}}}{8.26 r_{\mathrm{s}}^{2}+r_{\mathrm{s}}^{3}}}
$$

is a parametrized function that guarantees that the ACFDT under this OHU kernel reproduces exactly the HEG correlation energy. Note that despite the differences between these two approximations, for two electron systems both kernels are rather similar. Henceforth, the $\mathrm{OHU}$ energy-optimized nonlocal kernel also takes into account the correction to self-interaction errors at least at the same level than the PGG kernel. 
TABLE I: ACFDT Correlation Energies per Electron (mHa) for Closed-shell Jellium Clusters Compared with DMQC (ref 54), LDA, and GGA

\begin{tabular}{|c|c|c|c|c|c|}
\hline \multicolumn{6}{|c|}{$N$ DQMC RPA + OHU PGG KS-LDA KS-GGA } \\
\hline \multicolumn{6}{|c|}{$r_{\mathrm{s}}=2$} \\
\hline 2 & -16.9 & -19.2 & $-17.6-19.6$ & -35.7 & -21.0 \\
\hline 8 & -26.1 & -26.7 & $-26.6-23.2$ & -39.1 & -29.7 \\
\hline 20 & -30.5 & -31.5 & $-31.3-27.3$ & -40.6 & -33.8 \\
\hline 34 & -33.1 & -33.6 & $-33.4-29.8$ & -41.5 & -35.6 \\
\hline 58 & -34.7 & -35.2 & $-35.0-31.5$ & -42.1 & -37.2 \\
\hline \multicolumn{6}{|c|}{$r_{\mathrm{s}}=3.25$} \\
\hline 2 & -15.3 & -17.4 & $-16.5-18.9$ & -30.2 & -19.2 \\
\hline 8 & -22.4 & -23.3 & $-23.0-21.1$ & -32.1 & -25.3 \\
\hline 20 & -26.0 & -26.7 & $-26.4-24.2$ & -32.9 & -28.1 \\
\hline 34 & -27.5 & -28.0 & $-27.7-25.7$ & -33.5 & -29.3 \\
\hline 58 & -28.5 & -29.0 & $-28.7-26.5$ & -33.8 & -30.4 \\
\hline \multicolumn{6}{|c|}{$r_{\mathrm{s}}=4$} \\
\hline 2 & -14.6 & -16.5 & $-15.8-18.5$ & -27.8 & -18.3 \\
\hline 8 & -20.8 & -21.7 & $-21.4-20.2$ & -29.2 & -23.4 \\
\hline 20 & -24.1 & -24.7 & $-24.4-22.9$ & -29.8 & -25.7 \\
\hline 34 & -25.1 & -25.8 & $-25.4-24.1$ & -30.3 & -26.7 \\
\hline 58 & -26.2 & -26.8 & $-26.6 \quad-25.1$ & -30.6 & -27.6 \\
\hline \multicolumn{6}{|c|}{$r_{\mathrm{s}}=5.62$} \\
\hline 2 & -13.1 & -15.3 & $-14.6-17.6$ & -24.0 & -16.5 \\
\hline 8 & -18.1 & -19.2 & $-18.9-18.7$ & -24.8 & -20.1 \\
\hline 20 & -20.6 & -21.5 & $-21.1-20.9$ & -25.1 & -21.8 \\
\hline 34 & -21.7 & -22.2 & $-21.9-21.6$ & -25.4 & -22.6 \\
\hline 58 & -22.2 & -22.7 & $-22.4-22.4$ & -25.6 & -23.2 \\
\hline
\end{tabular}


In Table I, the obtained jellium clusters ACFDT correlation energies (RPA+, OHU, and PGG) are compared with the quasi-exact fixed-node diffusion quantum Monte Carlo (DQMC) results by Sottile and Ballone. ${ }^{54}$ For completeness and despite their low quality, the KS-LDA and KS-GGA correlation energies are included as well. ${ }^{67}$ The very good performance of the RPA + for all densities and electron numbers is evident, since the absolute errors are merely of the order of $1 \mathrm{mHa}$ per electron, except in the limit of $N=2$, where this error is slightly greater $(2-3 \mathrm{mHa} / \mathrm{e})$. In general, the more the number of electrons the smaller the relative errors committed by the RPA+, since the LDA/GGA correction term $\Delta E_{\mathrm{C}}$ in $(6)$ guarantees the exact reproduction of the HEG correlation energy. However, bearing in mind the simplicity of the RPA+ scheme, the good results for small and medium size clusters $(N=8,20)$ is rather streaking since these clusters are far from the homogeneous limit. The PGG does not provide any systematic improvement upon the RPA+ results and, in fact, the correlation energies are worse for two electron clusters. For large and medium size clusters, the slightly poor overall performance of the PGG-ACFDT with respect to the RPA+ is not a surprise, since the PGG-ACFDT energies are not exact in the homogeneous limit. ${ }^{26}$ However, the bad performance of the PGG-ACFDT for two electron systems (3-5 mHa/e deviations from the DQMC energies) is somehow disappointing, showing that the use of the exact exchange-only kernel is not enough to obtain accurate correlation energies. Finally, the use of the nonlocal OHU kernel, where both exchange and correlation contributions to the kernel are incorporated in an approximate manner, provides remarkably good results for all densities and electron numbers, including the problematic $N=2$ clusters. Although numerically a bit more involved than $\mathrm{RPA}+$, the $\mathrm{OHU}$ prescription truly corresponds to an implementation of the TDDFT to evaluate interacting density responses. Furthermore, the correlation energies are quite robust with respect to the details of the optimized kernel. ${ }^{32}$ These results confirm the superiority of schemes based on nonlocal approximations to the XC kernel for ACFDT correlation energy calculations. ${ }^{68}$

The above correlation energies have been evaluated using LDA Kohn-Sham wave functions. In principle this would be a bad choice since, as it is well known, the KS effective potential behaves as $-1 / r$ for $r \gg 0$ in localized systems. Therefore, all the series of Rydberg unoccupied orbitals are missed in the KS-LDA and, moreover, the LDA orbital eigenenergies themselves are not very accurate. This explains why the inclusion of EXX effects in the KS equations is mandatory to obtain realistic optical properties through the TDDFT. However, 
we have checked the choice of the reference KS systems is not critical when calculating ACFDT correlation energies, since they arise after a sum over all unoccupied states matrix elements and after integrations over all imaginary frequencies. In fact, if the EXX/LDA wavefunctions are used (i.e. the wavefunctions are evaluated selfconsistently using the EXX functional and a LDA prescription for the correlation), the ACFDT correlation energies only suffer changes always less than $0.2 \mathrm{mHa} / \mathrm{e} .{ }^{69}$ We can conclude that, contrary as occurs in the evaluation of optical properties of low-dimensional systems, the ACFDT results are extremely robust with respect to the choice of KS functions and energies. In other words, spectral details that are crucial in the obtention of TDDFT optical properties are not so important in the evaluation of integrated quantities like $E_{\mathrm{C}}$.

\section{CONCLUSIONS}

In this paper we have presented extensive results for the correlation energy of manyelectron systems using a sophisticated approach (ACFDT) based on the knowledge of the interacting response function. This ACFDT prescription accounts for many-body effects that are absent in standard implementations of the Kohn-Sham density functional theory. This methodology is much more expensive than LDA/GGA, but physically well motivated approximations within the ACFDT scheme provides correlation energies very close to the exact (up to numerical convergence) configuration interaction or quantum Monte Carlo results at a lower numerical cost.

For extended systems, we have explained in detail the numerical implementation of the ACFDT scheme thus paving the way to further studies specially aimed for situations where the standard LDA-GGA lacks predictive accuracy. On the other hand, several ACFDT approximations have been carefully assessed in a family of model systems (jellium clusters). Superior results are obtained if the XC kernel that enters into the evaluation of the response function is modelled in a nonlocal fashion. We have also checked that the ACFDT results are rather insensitive to the KS wavefunctions used to obtain the response function. Henceforth, for extended systems it is safe to evaluate the ACFDT correlation energies using LDA or GGA wavefunctions. For localized systems the method of choice should be EXX/LDA, but due to the accuracy required in the exact exchange energy since the ACFDT correlations are practically the same if we use LDA wavefunctions instead. 
Some issues need to be addressed in the future. First it is still not clear what is the optimal pseudopotential for EXX/ACFDT calculations. Certainly, a full EXX/ACFDT-based construction of the pseudopotential should be the proper way, and calculations along this line are in the horizon. Second, more efficient numerical implementations are required. For instance, much of the computational effort is employed to calculate contributions from highenergy unoccupied states that could be effective modelled by free-electron planewaves. Work in this direction is presently in progress as well. Finally, ongoing selfconsistent implementations of the EXX/ACFDT scheme will have a clear impact in the evaluation of many other properties besides structural ones. Highly accurate KS wavefunctions and eigenergies are required for reliable predictions regarding optical properties, phonons, etc. in low-dimensional systems (for instance, conjugate polymers). ${ }^{70}$

\section{Acknowledgment}

The authors thank J. E. Alvarellos, J.F. Dobson, J. Fernández-Rico, R. W. Godby, J. Jung, and M. Grüning for fruitful discussions. This work has being funded by the EC Network of Excellence NANOQUANTA (ref. NMP4-CT-2004-500198), the Spanish Ministry of Education (grant FIS2004-05035-C03-03), the SANES (ref. NMP4-CT-2006-017310), DNA-NANODEVICES (ref. IST-2006-029192), and NANO-ERA Chemistry projects, the University of the Basque Country EHU/UPV (SGIker Arina), and the Basque Country Government. The research by JJF and PGG is financed by the Juan de la Cierva and the Ramon y Cajal Programs, respectively. We thankfully acknowledge the computer resources, tech-

nical expertise and assistance provided by the Barcelona Supercomputing Center - Centro Nacional de Supercomputación.

\section{References and Notes}

1 Kohn, W.; Sham, L. J. Phys. Rev. 1965, 140, A1133.

2 Hohenberg, P.; Kohn, W. Phys. Rev. 1964, 136, B864.

3 Becke, A. D. Phys. Rev. A 1988, 38, 3098.

4 Perdew, J. P.; Burke, K.; Ernzerhof, M. Phys. Rev. Lett. 1996, 77, 3865. 
5 Alonso, J. A.; Girifalco, L. A. Phys. Rev. B 1978, 17, 3735.

${ }^{6}$ Gunnarsson, O; Jonson, M.; Lundqvist, B. I. Phys. Rev. B 1979, 20, 3136.

7 Chacón E.; Tarazona, P. Phys. Rev. B 1988, 37, 4014.

8 Alonso, J. A.; Cordero, N. A. In Recent Developments and Applications of Modern Density Functional Theory; Seminario, J. M., Ed.; Elservier: Amsterdam, 1996.

9 García-González, P.; Alvarellos, J. E.; Chacón E.; Tarazona, P. Phys. Rev. B 2000, $62,16063$.

10 Tao, J.; Perdew, J. P.; Staronerov V. N.; Scuseria, G. E. Phys. Rev. Lett. 2003, 91, 146401.

11 Staroverov, V. N.; Scuseria, G. E.; Tao, J.; Perdew, J. P. Phys. Rev. B 2004, 69, 075102 .

12 Constantin,L. A.; Perdew, J. P.; Tao, J. Phys. Rev. B 2006, 73, 205104.

13 Görling A.; Levy, M. Phys. Rev. A 1994, 50, 196.

14 Engel, E.; Facco Bonetti, A.; Keller, S.; Andrejkovics, I.; Dreizler, R. M. Phys. Rev. A 1998, 58, 964 .

15 Engel, E.; Jiang, H. Int. Journal of Quantum Chemistry 2006, 106, 3242.

16 Langreth, D. C.; Perdew, J. P. Solid State Commun. 1975, 17, 1425.

17 Langreth, D. C.; Perdew, J. P. Phys. Rev. B 1977, 15, 2884.

18 Runge, E.; Gross, E. K. U. Phys. Rev. Lett. 1984, 52, 997.

19 Time-Dependent Density Functional Theory; Marques, M. A. L.; Ulrich, C. A.; Nogueira, F.; Rubio, A.; Burke K.; Gross, E. K. U., Eds.; Springer: Berlin, 2006.

20 Onida, G.; Reining, L.; Rubio, A. Rev. Mod. Phys. 2002, 74, 601.

21 Hult, E.; Andersson, Y.; Lundqvist, B. Phys. Rev. Lett. 1996, 77, 2029.

${ }^{22}$ Kohn, W.; Meir, Y.; Makarov, D. E. Phys. Rev. Lett. 1998, 80, 4153. 
23 Dobson, J. F.; McLennan, K.; Rubio, A.; Wang, J.; Gould, T.; Le, H. M.; Dinte, B. P. Aust. J. Chem. 2002, 54, 513.

24 Rydberg, H.; Lundqvist, B. I.; Langreth, D. C.; Dion, M. Phys. Rev. B 2000, 62, 6997.

25 Rydberg, H.; Dion, M.; Jacobson, N.; Schröder, E.; Hyldgaard, P.; Simak, S. I.; Langreth, D. C.; Lundqvist, B. I. Phys. Rev. Lett. 2003, 91, 126402.

26 Lein, M.; Gross, E. K. U.; Perdew, J. P. Phys. Rev. B 2000, 61, 13431.

27 Kurth, S.; Perdew, J. P.; Phys. Rev. B 1999, 59, 10461.

28 Yan, Z.; Perdew, J. P.; Kurth, S. Phys. Rev. B 2000, 61, 16430.

29 Furche, F.; Voorhis, T. J. Chem. Phys. 2005, 122, 164106.

30 Dobson, J. F.; Wang, J. Phys. Rev. B 2000, 62, 10038.

31 Pitarke, J. M.; Perdew, J. P. Phys. Rev. B 2003, 67, 045101.

32 Jung, J.; García-González, P.; Dobson, J. F.; Godby, R. W. Phys. Rev. B 2004, 70, 205107.

33 Niquet, Y.-M.; Fuchs, M.; Gonze, X. Phys. Rev. A 2003, 68, 032507.

34 Niquet, Y.-M.; Fuchs, M.; Gonze, X. J. Chem. Phys. 2003, 118, 9504.

35 Niquet,Y.-M.; Gonze, X. Phys. Rev. B 2004, 70, 245115.

36 Talman, J. D.; Shadwick, W. F. Phys. Rev. A 1976, 14, 36.

37 For a review on exact exchange methods see, for instance: Engel, E. In A Primer on Density Functional Theory; Fiolhais, C.; Nogueira, F.; Marques M. A. L., Eds.; Springer: Berlin, 2003.

38 Görling, A. Phys. Rev. Lett. 1999, 83, 5459.

39 Sala, F. D.; Görling, A. J. Chem. Phys. 2001, 115, 5718.

40 Städele, M.; Majewski, J. A.; Vogl, P. ; Görling, A. Phys. Rev. Lett. 1997, 79, 2089.

41 Horowitz, C. M.; Proetto, C. R.; Rigamonti, S. Phys. Rev. Lett. 2006, 57, 14974. 
42 Eguiluz, A. G.; Heinrichsmeier, M.; Flezsar, A.; Hanke, W. Phys. Rev. Lett. 1992, 68, 1359.

43 Fernández, J. J.; García-González, P. (unpublished).

44 Hellgren, M.; von Barth, U. cond-mat/0703819 2007.

45 Grüning, M.; Marini, A.; Rubio, A. J. Chem. Phys 2006, 124, 154108.

46 Dobson,J. F.; Wang, J. Phys. Rev. Lett. 1999, 82, 2123.

47 Pitarke, J. M.; Eguiluz, A. G. Phys. Rev. B 2001, 63, 45116.

48 Furche, F. Phys. Rev. A 2001, 64, 195120.

49 Fuchs, M.; Gonze, X. Phys. Rev. B 2002, 65, 235109.

50 Aryasetiawan, F.; Miyake, T.; Terakura, K. Phys. Rev. Lett. 2002, 88, 166401; 2003, 90, 189702 .

51 Fuchs, M.; Burke, K.; Niquet, Y.-M.; Gonze, X. Phys. Rev. Lett. 2003, 90, 189701.

52 Miyake, T.; Aryasetiawan, F.; Kotani, T.; van Schilfgaarde, M.; Usuda, M.; Terakura, K. Phys. Rev. B 2002, 66, 245103.

53 Marini, A.; García-González, P.; Rubio, A. Phys. Rev. Lett. 2006, 96, 136404.

54 Sottile, F.; Ballone, P. Phys. Rev. B 2001, 64, 045105.

55 Trouillier, N.; Martins, J. L. Phys. Rev. B 1991, 43, 1993.

56 See: Gonze, X.; Beuken, J.-M.; Caracas, R.; Detraux, F.; Fuchs, M.; Rignanese, G.M.; Sindic, L.; Verstraete, M.; Zerah, G.; Jollet, F.; Torrent, M.; Roy, A.; Mikami, M.; Ghosez, Ph.; Raty, J.-Y.; Allan, D. C. Comput. Mater. Sci. 2002, 25, 478.

57 Marini M; et al, the SELF Project: http://www.fisica.uniroma2.it/〜 self/.

58 Marini, A., PhD dissertation, University of Rome "Tor Vergata": 2002.

59 Godby, R. W.; Sham, L. J.; Schlüter, M. Phys. Rev. Lett. 1986, 56, 2415.

60 Godby, R. W.; Sham, L. J.; Schlüter, M. Phys. Rev. B 1987, 36, 6497. 
61 Sham, L. J.; Schlüter, M. Phys. Rev. Lett. 1983, 51, 1888.

62 Sham, L. J.; Schlüter, M. Phys. Rev. B 1985, 32, 3883.

63 von Barth, U.; Dahlen, N. E.; van Leeuwen, R.; Stefanucci, G. Phys. Rev. B 2005, 72, 235109 .

64 Brack, M. Rev. Mod. Phys. 1993, 65, 677.

65 Ekardt, W.; Shöne, W. D.; Pacheco, J. M. In Metal Clusters; Ekardt, W., Ed.; John Wiley ; Sons: Chichester, 1999.

66 Petersilka, M.; Gossmann, U. J.; Gross, E. K. U. Phys. Rev. Lett. 1996, 76, 1212.

67 Note that due to the cancellation of errors, LDA exchange-correlation energies are very accurate, specially for large clusters. For $N=2$ and $N=8$, however, the LDA performance is poorer.

68 Dobson, J. F. Lec. Notes. Phys. 2006, 706, 443.

69 However, and specially for $N=2$, the exact exchange energy changes if we consider either LDA or EXX/LDA wavefunctions. Nevertheless, these changes in the EXX energy are compensated by variation in the electrostatic and kinetic energies and the method followed in ref 54 to extract the correlation energy from DQMC total energies is perfectly valid.

70 Varsano, D.; Marini, A.; Rubio, A. (unpublished). 